\title{
Depressão em crianças: Uma breve revisão de literatura
}

\author{
Depression in children: A brief review of literature
}

\author{
Soraia Schwan ${ }^{[a]}$, Vera Regina Rohnelt Ramires ${ }^{[\mathrm{b}]}$ \\ [a] Mestre em Psicologia Clínica pela Universidade do Vale do Rio dos Sinos (UNISINOS), São Leopoldo, RS - Brasil, e-mail: \\ soraiaschwan@gmail.com \\ [b] Doutora em Psicologia Clínica pela Universidade do Vale do Rio dos Sinos (UNISINOS), São Leopoldo, RS - Brasil, e-mail: \\ vramires@unisinos.br
}

\begin{abstract}
Resumo
O quadro depressivo tem sido apontado, por diferentes autores, como sendo grave e comum a muitas crianças e adolescentes. Pode se caracterizar como um estado afetivo normal, um conjunto de sintomas ou até mesmo compreender uma síndrome ou doença específica. Com o objetivo de realizar uma revisão não sistemática de literatura sobre o tema da depressão em crianças, foram consultadas as principais bases de dados disponíveis on line, no período compreendido entre 2000 a 2010. Foram selecionados artigos que abordavam tópicos como: "aspectos epidemiológicos e sintomáticos", "comorbidades", "avaliação, diagnóstico e intervenções", "aspectos psicodinâmicos" e, ainda, "depressão e acolhimento institucional". Foi possível constatar que diferentes autores concordam acerca da gravidade da depressão na infância e apontam a importância de se desenvolverem estudos que contribuam com intervenções adequadas a essa população. Também foi identificada a concentração de diferentes produções científicas que buscam identificar a sintomatologia da depressão na infância e sua prevalência, mas ainda são raros os estudos que se voltam às intervenções, sobretudo, àquelas psicanaliticamente orientadas. De igual forma, são raros os estudos que buscam investigar a eficácia dos tratamentos para a depressão em crianças e adolescentes.
\end{abstract}

Palavras-chave: Autonomia. Professor. Adolescência.

\begin{abstract}
The depression has been suggested by different authors as being serious and common to many children and adolescents. Can be characterized as a normal affective state, a set of symptoms or even understand a specific disease or syndrome. Aiming to achieve a non-systematic review of literature on the topic of depression in children have been consulted major databases available online, in the period 2000-2010. We selected articles that addressed topics such as "symptomatic and epidemiological aspects", "comorbidities", "evaluation, diagnosis and interventions" "psychodynamic", and "depression and residential care". It was noted that different authors agree about the severity
\end{abstract}


of depression in childhood and highlight the importance of developing studies that contribute to appropriate interventions for this population. They also identified the concentration of various scientific studies that seek to identify the symptoms of depression in childhood and its prevalence, but there are few studies that focus on the interventions, especially those psychoanalytically oriented. Similary, there are few studies that attempt to investigate the efficacy of treatments for depression in children and adolescents.

Keywords: Depression. Children. Literature review.

\section{Introduçáo}

O objetivo deste estudo foi realizar uma revisão não sistemática de literatura sobre o tema da depressão em crianças. Foram consultadas as bases de dados BVS (SciELO, LILACS, MEDLINE), PsycINFO e Academic Search Premier. Os descritores utilizados inicialmente foram "depression" e "children". Em razão do expressivo número de produções identificadas (mais de 16 mil), sendo muitas delas não específicas sobre o foco de interesse, utilizou-se a expressão “depression in children". O período abrangido pela revisão foi o de 2000 a 2010, selecionando-se para análise artigos que abordavam os seguintes tópicos: "aspectos epidemiológicos e sintomáticos", "comorbidades", "avaliação, diagnóstico e intervenções", "aspectos psicodinâmicos" e, ainda, "depressão e acolhimento institucional".

Constatou-se que a literatura vem reconhecendo a importância e a presença dos quadros depressivos na infância (Bahls, 2002; Bahls \& Bahls, 2003; Calderaro \& Carvalho, 2005; Cruvinel \& Boruchovitch, 2003; Dutra, 2001; Fensterseifer \& Werlang, 2003). A relevância desse reconhecimento é evidente, se considerada a necessidade de intervenção adequada e de prevenção da cronificação e de problemas posteriores.

Sobre a definição da depressão na infância, o Manual Diagnóstico e Estatístico de Transtornos Mentais (American Psychiatric Association - APA, 2000) não diferencia os critérios diagnósticos dos transtornos depressivos para crianças, adolescentes ou adultos. Tais transtornos estão inseridos, nesse Manual, no grupo dos transtornos do humor, assim como os transtornos bipolares. São descritos nos quadros do transtorno depressivo maior, do transtorno distímico e do transtorno depressivo sem outra especificação.

O transtorno depressivo maior caracteriza-se por um ou mais episódios depressivos maiores: duas semanas de humor deprimido ou perda de interesse acompanhado por pelo menos quatro dos seguintes sintomas: diminuição ou aumento do apetite, falha em apresentar os ganhos de peso esperados para a idade, insônia ou hipersonia, agitação ou retardo psicomotor, fadiga ou perda de energia, sentimento de inutilidade ou culpa excessiva, dificuldade de concentração, pensamentos de morte recorrentes, ideação suicida ou tentativa de suicídio (APA, 2000). O transtorno distímico é caracterizado por humor deprimido ou irritável por pelo menos um ano e pelo menos duas das seguintes características: apetite diminuído ou hiperfagia, insônia ou hipersonia, baixa energia ou fadiga, baixa autoestima, dificuldade de concentração. O transtorno depressivo sem outra especificação inclui transtornos com características depressivas que não satisfazem os critérios anteriores.

Caracterizada, portanto, como um transtorno de humor, a depressão abrange fatores cognitivos, comportamentais, fisiológicos, sociais e econômicos, entre outros (Ribeiro, Oliveira, Coutinho \& Araújo, 2007). Lima (2004) aponta que o conceito de depressão na infância ainda é muito controverso e que não pode ser confundido com o sentimento de tristeza, já que este faz parte da experiência normal do sujeito. Para Wathier e Dell'Aglio (2007) o termo depressão tem sido designado em diferentes amplitudes, que podem caracterizar um estado afetivo normal, a apresentação de um conjunto de sintomas ou até mesmo pode compreender uma síndrome ou doença específica.

Nessa direção, a Organização Mundial da Saúde - OMS (1993) já havia sinalizado na década de 1990 que a depressão implica aspectos biológicos e psicológicos e que o prognóstico para esse quadro pode ser bastante negativo em alguns casos. A estimativa para 2020, segundo a OMS, é de que a depressão seja a maior causa de incapacidade humana, somente sendo superada pelas doenças cardiovasculares. 
Os quadros depressivos na infância têm sido descritos há bastante tempo (Reis \& Figueira, 2001). Entretanto, foi somente a partir da década de 1960 do século passado que as investigações começaram a ser sistematizadas, para Baptista e Golfeto (2000). Já para Bahls (2002), Reis e Figueira (2001) e Scivoletto e Tarelho (2002), o quadro depressivo na infância despertou interesse na sistematização de investigações mais detalhadas a partir da década de 1970, depois que muitos profissionais de saúde constataram que essa patologia trazia comprometimentos importantes nas funções sociais, emocionais e cognitivas, interferindo no desenvolvimento infantil. Com o reconhecimento da depressão em crianças e adolescentes pelo Instituto Nacional de Saúde Mental dos Estados Unidos, houve um avanço de produções que focalizavam a avaliação e o diagnóstico dessa patologia na infância, segundo Bahls (2002).

\section{Sobre os aspectos epidemiológicos e sintomáticos}

Na população brasileira, estimativas apontam que de $0,4 \%$ a $3 \%$ das crianças apresentam características depressivas (Bahls, 2002). Em adolescentes, esse número varia de 3,3 a 12,4\%, com fortes indicativos de se desenvolver a doença em idade adulta (Bahls \& Bahls, 2003). Em crianças abrigadas, aquelas que sofreram conflitos e rompimentos de vínculos importantes, autores como Dell'Aglio e Hutz (2004) e Wathier e Dell'Aglio (2007) apontam a prevalência de $6 \%$ a $10 \%$ para a depressão nessa população. Já nos Estados Unidos, os índices sinalizam um percentual que varia de 3\% a 5\% (Goldberg, 2007). Em crianças australianas, o índice de prevalência da depressão encontrado por Hazell (2009) é de $2 \%$ a 8\%; e na Alemanha, segundo Mehler-Wex e Kölch (2008), estima-se que a prevalência da depressão em crianças e adolescentes seja de 8,9\%.

Alguns estudos apontam a depressão na infância como um problema crescente (Dell'Aglio \& Hutz, 2004; Ramires, Passarini, Flores \& Santos, 2009; Zavaschi et al, 2002). Muitos buscam descrever características demográficas e a sintomatologia (Bahls, 2002; Kessler, Avenevoli \& Mericangas, 2001; Wathier \& Dell'Aglio, 2007). Os principais sintomas descritos pelos estudos são: transtorno por déficit de atenção e hiperatividade, baixa autoestima, medos, distúrbios do sono, enurese, tristeza, dores abdominais, culpa, fadiga, desinteresse por atividades de modo geral, ideação suicida (Calderaro \& Carvalho, 2005) e problemas de aprendizagem (Cruvinel \& Boruchovitch, 2003; Dell'Aglio \& Hutz, 2004). As causas são relacionadas, na maioria dos estudos, aos aspectos psicossociais, ou seja, perda de vínculos afetivos, divórcio dos pais, violência física e psicológica, falta de apoio familiar, (Bahls, 2002; Calderaro \& Carvalho, 2005; Dell'Aglio, Borges \& Santos, 2004; Lima, 2004; Ramires et al., 2009b).

Zavaschi et al., em um estudo publicado em 2002, associaram as perdas da infância à depressão na vida adulta, e as crianças que sofreram rompimentos vinculares significativos tenderam ao desenvolvimento da patologia depressiva na sua vida adulta. As perdas estavam relacionadas à história de separação dos pais, porém, não à morte deles.

A queda do rendimento escolar é um dos primeiros indicadores de depressão em crianças, além do desenvolvimento do quadro de disforia, isolamento e tristeza. Os sintomas da depressão também são considerados, por diferentes autores, como preditores de outras patologias e, nesse caso, estão associados às comorbidades. Tais comorbidades nem sempre são diagnosticadas como preliminares ao desenvolvimento do quadro depressivo. Muitas vezes aparecem como consequência. Além disso, alguns sintomas podem ser "recombinados" entre si, implicando dificuldades de diagnosticar a depressão na infância. Sintomas como apatia, perda de peso, recusa escolar e apetite diminuído, apresentam relação de dependência para certos diagnósticos, podendo ser importantes para diagnósticos específicos (Bahls, 2002).

Embora os critérios diagnósticos sejam os mesmos dos adultos, nas crianças com transtorno depressivo maior há o predomínio de sintomas como ansiedade, queixas somáticas e alucinações. Em adolescentes, são comuns as alterações do sono e do apetite. Contudo, Versiani, Reis e Figueira (2000) apontam que a ideação suicida e as próprias tentativas de suicídio aparecem como agravamento dos sintomas. No diagnóstico de pacientes com transtorno distímico, são mais comuns os sentimentos de rejeição, raiva, comportamento desobediente, maior dificuldade social e maior prejuízo nas atividades de lazer.

Coutinho e Ramos (2008) afirmam que o quadro depressivo em crianças está associado a perturbações do humor de duração variáveis. Em geral, esse quadro é acompanhado por sintomas físicos e 
mentais, o que interfere na forma de sentir, pensar e agir dessa população.

Segundo Bahls (2002) e Bahls e Bahls (2003), a depressão na infância está aumentando e ocorrendo cada vez mais cedo. No entanto, muito embora se reconheça a depressão infantil como uma patologia, e apesar dela ser avaliada como um dos problemas mais frequentes na prática clínica (Rodríguez de Cossío \& Granada Jiménez, 2007), considera-se que ela tem sido pouco diagnosticada e, em consequência disso, poucos pacientes recebem tratamento. Scivoletto e Tarelho (2002) referem que o diagnóstico por vezes é dificultado em função das comorbidades, muitas vezes presentes nos quadros depressivos. Por conta disso, os sintomas se manifestam de forma não clara, inviabilizando ou dificultando o reconhecimento desse quadro em crianças.

Reis e Figueira (2001) também relataram que muitas vezes as próprias crianças têm dificuldades em identificar e nomear seus sintomas, que aparecem de maneira multifacetada. Por outro lado, os pais e os profissionais acabam identificando problemas que, inicialmente, não são reconhecidos como sendo de depressão, já que existem queixas orgânicas como cefaleia, dores abdominais e diarreia, que dificultam o diagnóstico referido.

Outra dificuldade encontrada no reconhecimento da depressão na infância ocorre porque não são comuns as distinções ou especificidades existentes na literatura entre a depressão infantil e aquela com manifestação na vida adulta. No entanto, vários autores afirmam que as particularidades da infância devem ser consideradas na avaliação e diagnóstico da depressão em crianças (Kuczynski, Marcolin \& Assunção Jr., 2001; Versiani, Reis \& Figueira, 2000).

\section{Depressáo na infância: Associaçóes e comorbidades}

Como dito anteriormente, as comorbidades ou sintomatologias associadas à depressão são difíceis de diagnosticar, principalmente em relação a se associarem como causa ou consequência da depressão na infância. O que muitos estudos vêm apontando é que crianças e adolescentes com transtorno depressivo apresentam uma condição clínica grave, crônica, recorrente e que é acompanhada por outros transtornos associados (Bahls,
2002). O diagnóstico implica o uso de diferentes critérios e a observação do relato da sintomatologia, conforme o nível de desenvolvimento da criança, como aponta a autora.

Os estudos que abordam as comorbidades em geral mencionam o déficit escolar, a obesidade na infância e doenças crônicas ou deficiências físicas, o que, consequentemente, agrava o estado clínico geral dos envolvidos (Castro, 2003). Transtornos de ansiedade (especialmente o transtorno de ansiedade de separação), o transtorno de conduta, o transtorno desafiador opositivo e o transtorno por déficit de atenção também são apontados por Bahls (2002).

Conforme afirma Castro (2003), crianças com doenças crônicas possuem maiores riscos de apresentar depressão, o que está relacionado com deterioração física, gravidade e modo de enfrentamento familiar relacionado à doença. Para Cabrera et al. (2005), as características de crianças com câncer e que apresentam baixos níveis de depressão foram associadas à forma defensiva de lidar com a ansiedade e os medos, o que também estaria relacionado ao aumento de sintomas físicos.

Os fatores de risco associados ao desenvolvimento da depressão infantil também são descritos pelas produções científicas que apontam como principais preditores: o embotamento afetivo dos pais (Abela, Skitch, Auerbach \& Adams, 2005); o uso precoce do uso de álcool (Auger, 2005); a presença do transtorno de déficit de atenção e hiperatividade (Calvillo, 2004); o transtorno de ansiedade (Chorney, Detweiler, Morris \& Kuhn, 2008); a rejeição dos colegas (bullying) apontado por Morrow et al. (2006) e por West e Salmon (2000); e o abuso físico (Finzi et al., 2001), somente para citar alguns.

Para Dell'Áglio e Hutz (2004), o rendimento escolar em crianças com sintomas depressivos parece bastante comprometido em relação ao das crianças na mesma faixa etária que não apresentam tal sintomatologia. As principais variáveis envolvidas no déficit escolar referem-se às funções cognitivas e afetivas, como a função mnemônica e a autoestima.

Grote e Frank (2003) relatam que a ocorrência de depressão associada a outros diagnósticos é realmente maioria e, por isso, consideram o papel da psicoterapia fundamental na abordagem desses pacientes. Esses autores apontam a necessidade de abordagem individualizada para o diagnóstico e tratamento da depressão. 


\section{Avaliaçáo, diagnóstico e possibilidades de intervençáo}

Para Abaid (2008), mesmo que a avaliação clínica seja a forma mais adequada de se diagnosticar ou analisar a presença de indicadores de depressão em crianças, alguns instrumentos podem auxiliar nessa investigação, como a Escala de Avaliação do Grau de Severidade da Depressão Infantil (CDRS-R), referida por Wagner et al. (2006); o instrumento Trauma Symptom Checklist for Children (TSC-C), referido por Margetic et al. (2005); o PictorialDepression Scale (PDS), apontado por O'Connor e Paley (2006); o Schedule for Affective Disorders and Schizophrenia (Kiddie - SADS), como referem Versiani, Reis e Figueira (2000); a Reynolds Adolescents Depression Scale (RADS), referida por Brajsa-Zganec em 2005; e o Children Depression Inventory (CDI), trazido por Kovács (2003) e mais recentemente por Wathier e Dell'Aglio (2007).

O CDI (Kovács, 1983, 1985, 1992, 2003) vem sendo descrito como o instrumento mais utilizado internacionalmente para avaliar sintomas depressivos em crianças e adolescentes, tanto nos contextos clínicos como de pesquisa (Baptista \& Golfeto, 2000; Cole et al., 2002). O objetivo desse instrumento é verificar a presença e a severidade de sintomas de depressão em jovens de 7 a 17 anos, com base no seu autorrelato, sendo adaptado do Inventário Beck de Depressão (BDI) para adultos. O CDI foi adaptado para uso no Brasil por Gouveia, Barbosa, Almeida e Gaião (1995), demonstrando características psicométricas adequadas, tendo sua consistência interna variado entre 0,79 e 0,92 (Dell'Aglio, Borges \& Santos, 2004; Reppold \& Hutz, 2003).

Posteriormente, suas propriedades psicométricas foram verificadas por Wathier, Dell'Aglio e Bandeira (2008), em uma amostra de 951 crianças e adolescentes, com idades entre 7 e 17 anos, da região Sul do Brasil. Seiscentos e trinta e quatro participantes viviam com suas famílias e 317 em instituições. As autoras encontraram uma composição trifatorial e um alfa de Cronbach de 0,85, evidenciando boa consistência interna.

Pode-se afirmar que existe um consenso na literatura apontando que a depressão na infância vem acompanhada por uma multiplicidade de fatores sociais, psicológicos e biológicos (Baptista \& Golfeto, 2000; Reppold \& Hutz, 2003; Wathier, Dell'Aglio, 2007). Essa multiplicidade de fatores deve ser levada em conta quando se realiza a avaliação da depressão em crianças. Os instrumentos podem serimportantes instrumentos de avaliação, porém, não substituem a avaliação clínica (Abaid, 2008).

Uma avaliação adequada pode conduzir à intervenção necessária, uma vez que, segundo Bahls e Bahls (2003), por constituir doença grave e que possui uma continuação ou recorrência na idade adulta, a abordagem terapêutica para a depressão na infância é de extrema importância. Entretanto, conforme Watanabe et al. (2007) e Fonagy et al. (2002), praticamente não existem estudos comparativos de eficácia entre as formas de psicoterapia empregadas no tratamento dessa população.

As psicoterapias são amplamente indicadas na depressão infanto-juvenil, tanto nos casos de intensidade sintomatológica leve a moderada como associada à psicofarmacologia nos casos mais graves (Son \& Kirchner, 2000). As abordagens mais utilizadas são: a terapia cognitivo-comportamental (Gladstone \& Bearsdslee, 2009), a psicoterapia de orientação psicodinâmica e a psicoterapia interpessoal (Ito \& Lotufo Neto, 2000), muitas vezes associadas aos psicofármacos (Jureidini, 2009; Varley, 2006). Algumas intervenções em formato individual, grupal e familiar (Horn et al., 2005) têm se mostrado bastante eficazes, porém, como apontam Horn et al., (2005), muitos pacientes necessitam de tratamento mais intensivo. As orientações psicoeducacionais também têm sido mencionadas como habitualmente empregadas e úteis (Ito \& Lotufo Neto, 2000). No entanto, poucos são os ensaios clínicos controlados existentes.

A técnica cognitivo-comportamental (com suas várias modalidades) tem sido amplamente empregada para a depressão. A melhora clínica na população adolescente nessa modalidade de intervenção apresentou resultados entre 50\% a 65\% (Bahls \& Bahls, 2003; Michael \& Crowley, 2002). Entretanto, de acordo com Weisz, McCarty e Valeri (2006), mesmo diminuindo os níveis de ansiedade, esse modelo de tratamento psicoterapêutico se mostrou ineficiente quando analisados os efeitos a longo prazo.

Segundo Horn et al. (2005), a psicoterapia psicanalítica mostra-se, já no curto prazo, um tratamento efetivo para crianças e adolescentes deprimidos. Embora essa modalidade psicoterápica tenha muito a contribuir nessa instância, ainda são necessários muitos estudos nessa área. Deakin e Nunes (2008) e Fonagy et al. (2002) e Fonagy (2003) propõem que o terapeuta sistematize suas impressões 
para poder analisar a adequação e a eficácia do tratamento psicanalítico. Isso seria importante, pois, conforme Fonagy (2003), a natureza do trabalho psicanalítico talvez seja incompatível com as características dos estudos controlados.

Bahls e Bahls (2003), em estudo intitulado "Psicoterapias da depressão na infância e na adolescência", citam a Task Force on Promotion and Dissemination of Psychological Procedures (1995), que assinala os indicadores importantes para os estudos em psicoterapia:

a) um modelo teórico coerente;

b) um manual de tratamento com métodos específicos de procedimento;

c) um formato de tempo limitado;

d) medidas específicas de avaliação de resultados. As psicoterapias que desejam integrar modelos científicos de tratamento obrigatoriamente necessitariam adequar-se a esses requisitos.

Fonagy et al. (2002) indicam que, em geral, os estudos sobre o tratamento do quadro depressivo em crianças e adolescentes demonstram uma adaptação de modelos adultos, o que deixa clara a falta de compreensão acerca do desenvolvimento, que deveria identificar condições próprias de cada etapa do crescimento emocional. Essa perspectiva da psicopatologia do desenvolvimento pode requerer desenhos de intervenção e de pesquisa específicos em diferentes momentos na infância e na adolescência. Além disso, esses autores acrescentam que ainda existe muito pouco conhecimento advindo de pesquisas sobre a importância do envolvimento dos pais e da família nos processos terapêuticos. Fonagy e seus colaboradores apontam que a maioria dos estudos se refere ao uso de medicamentos ou situa as intervenções em propostas cognitivo-comportamentais. Entretanto, em que pese a eficácia dessa modalidade de intervenção nos quadros de depressão, esses autores assinalam que a terapia cognitivo-comportamental é menos efetiva nos casos mais severos de depressão e naqueles em que há maiores distorções cognitivas.

A literatura internacional, assim como a brasileira, é restrita no que diz respeito à investigação de intervenções adequadas para o tratamento da depressão infantil. Tem focalizado mais o tratamento com adultos, assim como o que diz respeito à análise da sintomatologia e das suas causas (Blatt-Eisengart,
Drabick, Monahan \& Steinberg, 2009; Lakdawalla, Hankin \& Mermelstein, 2007; Perera, 2008; Suveg, Hoffman, Zeman \& Thomassin, 2009; Wasliick, Schoenholz \& Pizarro, 2003).

Um recente estudo piloto realizado por Fischer-Kern et al. (2008) investigou a capacidade de mentalização em pacientes depressivos. Esse estudo analisou a capacidade de mentalização em 20 pacientes do sexo feminino que sofriam de transtorno depressivo maior, utilizando instrumentos como o Skidi + II, Escalas de Depressão (HAM-D, BDI), testes cognitivos (TMT, MCVCT) e a Adult Attachment Interview (AAI). Os resultados foram avaliados por meio da Escala da Função Reflexiva aplicada a AAI e revelaram que os pacientes deprimidos apresentam menor capacidade de mentalização do que os pacientes borderline e os indivíduos saudáveis. Foi constatado também que há uma variação da capacidade de mentalização, conforme a gravidade da depressão. Diante desses dados, os autores concluíram que há necessidade de estudos que investiguem as mudanças na capacidade de mentalização de pacientes com depressão, no curso dos tratamentos psicoterápicos.

\section{Aspectos psicodinâmicos relacionados à depressáo}

Vivências de perdas têm sido associadas na literatura aos quadros de depressão na infância. Franco e Mazorra (2007) analisaram crianças que sofreram perdas significativas na infância, relativas aos genitores, e concluíram que essas crianças apresentavam fantasias de aniquilamento, culpa, castração, onipotência, rejeição, identificação, retaliação, idealização e desidealização do objeto perdido, além de agressividade, negação da perda, regressão, reparação e repetição da situação da perda. As autoras ainda apontaram que, diante da perda significativa do vínculo provedor de sustentação, a criança se depara com profundos sentimentos de desamparo e impotência. Embora as autoras se refiram às perdas por morte dos genitores, seu estudo contribui para a elucidação dos quadros depressivos apresentados por crianças e relacionados à perda ou separação dessas figuras.

O estudo de Franco e Mazorra (2007) foi orientado pelas contribuições de Freud (1996) e Bowlby (1989) para explicar o processo de en- 
lutamento de crianças que sofreram perdas significativas. Para Bowlby (1989), enquanto a criança não pode reconhecer seus objetos de amor como sendo separados dela, na eventualidade de perda ou separação da mãe, ela sente que perdeu uma parte de si própria e, conforme apontam Franco e Mazorra (2007), o enlutamento por si só não pode dar conta da perda do objeto amado. Nessa mesma direção, Trevisan (2004) abordou a questão da depressão em crianças que tiveram perdas significativas na infância, associando-a à melancolia, que implica uma regressão para a fase oral narcisista da libido, em que ocorre uma identificação com o objeto perdido. Em consequência disso, o ego sofre com a autotortura, observada clinicamente, e que expressa uma tendência ao sadismo e ao ódio relacionados ao objeto e que retornam ao próprio eu do indivíduo.

Calderaro e Carvalho (2005) e Rotondaro (2002) salientaram a necessidade de um ambiente favorável ao desenvolvimento emocional, supridor de necessidades básicas como proteção e acolhimento. Por outro lado, quando esse ambiente desfavorece a criança em suas necessidades, ela se utiliza de mecanismos de defesa específicos para lidar com as dificuldades, muitas vezes desenvolvendo um quadro depressivo. Consequentemente, a estrutura de sua personalidade se vê comprometida,estrutura que durante a infância está em pleno desenvolvimento.

Calderaro e Carvalho (2005) acrescentam que a depressão na criança interfere em atividades fundamentais da sua vida e nas fases de seu desenvolvimento. Por conta disso, é muito importante o diagnóstico precoce, além da efetivação de medidas de promoção da saúde mental, que são fundamentais, uma vez que a criança depressiva se envolve em situações que oferecem perigo à sua integridade física, colocando-se em risco. Angerami-Camon (2002), nessa mesma linha, pontua que as crianças também se desesperam diante das dificuldades que encontram em suas vidas e manifestam o desejo de morrer. Essas manifestações, muitas vezes, podem estar relacionadas ao suicídio na infância e requerer a atenção e sensibilidade dos profissionais, especialmente no tocante ao diagnóstico e tratamento mais adequado.

Quanto aos fatores de risco para o desenvolvimento do funcionamento depressivo, Bahls e Bahls (2003) apontaram a preponderância da presença de depressão em um dos pais, seguida de fatores como a perda significativa de um dos genitores, estressores ambientais e abuso físico e violência. Esses aspectos nos levam a considerar a problemática da depressão e do acolhimento institucional.

\section{Depressão e acolhimento institucional}

A presença de violência física e/ou psicológica, de negligência, entre outros estressores ambientais, pode levar à necessidade do acolhimento institucional da criança. A depressão pode ser apresentada por essas crianças, embora haja controvérsias na literatura quanto aos seus escores relacionados ao sexo da criança. Alguns estudos apontam que não existem diferenças significativas nos escores de depressão em relação ao sexo das crianças que vivem em instituições (Bailey, Zauszniewski, Heinzer \& Hemstrom-Krainess, 2007; Kim \& Cicchetti, 2006). Por outro lado, Cole et al. (2002) afirmam ter encontrado diferenças significativas nessa população, utilizando o CDI, indicando que as meninas, em geral, possuem maiores escores de sintomatologia depressiva.

O afastamento do núcleo familiar pode produzir o rompimento dos vínculos afetivos com os objetos primários e incidir negativamente sobre o desenvolvimento saudável na infância (Alexandre \& Vieira, 2004). Isso é preocupante, uma vez que um número significativo de crianças se encontra nessa situação no Brasil. Silva, em artigo publicado em 2004, apontou um levantamento em abrigos para crianças e adolescentes em âmbito nacional, constatando que cerca de 20 mil crianças e adolescentes viviam em 589 abrigos no Brasil, estando a maior parte situada na região Sudeste, que concentrava 45\% dos abrigados. São Paulo comportava um terço dessa população. Nas demais regiões do Brasil mantinham-se altos índices de acolhimento institucional. O Nordeste, com 29,4\% dos abrigados, apresentava o maior índice entre as demais regiões. OSul concentrava 15,5\% das crianças abrigadas e o Centro-Oeste e Norte apresentavam, como porcentagem, $8,2 \%$ e $1,9 \%$, respectivamente. Somente $10,7 \%$ dessas crianças estavam judicialmente em condições de adoção, conforme Silva.

Estima-se que oito milhões de crianças no mundo estejam sob regime de acolhimento institucional (Wathier \& Dell'Aglio, 2007). A maioria delas estaria afastada de seus lares em razão da violência e desintegração familiar, além das situações econômicas e sociais não adequadas. 
Para Dell'Aglio e Hutz (2004), crianças que se encontram em condições desfavoráveis, como abandono e maus-tratos, e que vivem longe da convivência familiar apresentam maior vulnerabilidade a outras questões decorrentes da pobreza, ao envolvimento com drogas e ao desemprego. Sobretudo, conforme Runyon, Faust \& Orvaschel, 2002, crianças que tenham experimentado a falta de apoio familiar ou o rompimento dos vínculos afetivos demonstram maiores chances de desenvolver o distúrbio depressivo.

O rompimento dos vínculos foi apontado por diversos autores como fator de risco para o desenvolvimento da depressão em crianças (Dell'Aglio, Borges \& Santos, 2004; Dell'Aglio \& Hutz, 2004; Runyon, Faust \& Orvaschel, 2002; Zavaschi, et al., 2002). A situação de acolhimento institucional pode, portanto, implicar duas vias de conflitos: o rompimento de vínculos propriamente dito, com o afastamento da família, muitas vezes sentido pela criança como traumático, e o conflito relacionado à dificuldade de formar novos vínculos sadios. A depressão pode resultar do trauma sofrido pelo rompimento vincular familiar e dificultar o estabelecimento de novos vínculos.

No que se refere aos contextos de desenvolvimento, familiar e institucional, alguns estudos têm relacionado o cuidado institucional nos anos iniciais a dificuldades de comportamento e de personalidade. No entanto, Dell'Aglio e Hutz (2004) e Wathier e Dell'Aglio (2007) apontam fatores que podem contribuir na modificação dos efeitos da institucionalização na infância. Entre esses fatores devem ser consideradas as razões para a separação da família, o tipo de relacionamento prévio com a mãe, a oportunidade de desenvolver relações seguras após a separação, a qualidade do cuidado oferecido, a idade da criança, a duração da institucionalização, o sexo e o temperamento da criança.

Wathier e Dell'Aglio (2007) investigaram a prevalência de depressão em crianças e adolescentes institucionalizados e concluíram que essa população reúne diversas características que corroboram a presença desse problema, uma vez que sofreram rompimentos de vínculos por terem sido negligenciadas ou vitimadas por violência física e psicológica. Assim, segundo as autoras, os índices de depressão em crianças em instituições de abrigo são significativos, e em meninas esse número é ainda maior. Esses resultados sinalizam a urgência de se desenvolver pesquisas e intervenções que abordem essa problemática, já que, nesses casos, as chances de crianças se tornarem adultos depressivos é bastante grande.

\section{Consideraçóes finais}

Ainda que haja concordância entre os autores acerca da importância e gravidade da depressão na infância, que pode predizer ou estar associada a outras patologias, ainda assim não existe um consenso sobre a definição ou conceitualização da depressão nessa faixa etária. A falta de tais definições coloca em dúvida, muitas vezes, seu diagnóstico, o que, consequentemente, dificulta o tratamento e a prevenção da cronificação do problema. Nesse sentido, é indiscutível a relevância de se empreender esforços que possam investigar sistematicamente a depressão infantil, principalmente em razão da ineficácia dos diagnósticos e ao fato de que poucos pacientes recebem tratamento adequado, tornando-se, assim, um grande desafio para a saúde pública.

É possível constatar a grande concentração de estudos que buscam investigar a identificação da sintomatologia da depressão e sua prevalência, mas ainda são raros os estudos que se voltam às intervenções, sobretudo, àquelas psicanaliticamente orientadas. Verificam-se as lacunas existentes também no que tange à eficácia dos tratamentos para a depressão em crianças e adolescentes, já que uma grande preocupação é a necessidade de compreender melhor as etapas desenvolvimentais, para que se possa adequar melhores recursos de avaliação e tratamento na infância. Também seria importante compreender mais aprofundadamente os contextos familiares, suas dificuldades e possibilidades. Constata-se, ainda, uma carência importante de estudos dedicados à discussão e ao desenvolvimento de estratégias de prevenção, bem como no sentido da elaboração de diretrizes políticas para o enfrentamento desse problema.

\section{Referências}

Abaid, J. L. W. (2008). Vivências adversas e depressão: Um estudo sobre crianças e adolescentes institucionalizados. Dissertação de Mestrado não publicada. Universidade Federal do Rio Grande do Sul, RS, Porto Alegre. 
Abela, J. R. Z., Skitch, S. A., Auerbach, R. P., \& Adams, P. (2005). The impact of parental borderline personality disorder on vulnerability to depression in children of affectively ill parents. Journal of Personality Disorders, 19(1), 68-83.

Alexandre, D. T., \& Vieira, M. L. (2004). Relação de apego entre crianças institucionalizadas que vivem em situação de abrigo. Psicologia em Estudo, 9(2), 207-217.

American Psychiatric Association - APA. (2000). Manual diagnóstico e estatístico dos transtornos mentais - DSM-IV. Porto Alegre: Artmed.

Angerami-Camon, V. (2002). Criança também se autodestrói. Viver Psicologia, 11(116), 14.

Auger, R. W. (2005). School-Based interventions for students with depressive disorders. Professional School Counseling, 8(4), 344-352.

Bahls, S. C. (2002). Aspectos clínicos da depressão em crianças e adolescentes. Jornal de Pediatria, 78(5), 359-366.

Bahls, S. C., \& Bahls, F. R. (2003). Psicoterapias da depressão na infância e na adolescência. Estudos de Psicologia, 20(2), 25-34.

Bailey, M. K., Zauszniewski, J. A., Heinzer, M. M., \& Hemstrom-Krainess, M. (2007). Patterns of depressive symptoms in children. Journal of Child and Adolescent Psychiatric Nursing, 20(2), 86-95.

Baptista, C. A., \& Golfeto, J. H. (2000). Prevalência de depressão em escolares de 7 a 14 anos. Revista de Psiquiatria Clínica, 27(5), 253-255.

Blatt-Eisengart, I., Drabick, D. A., Monahan, K. C., \& Steinberg, L. (2009). Sex differences in the longitudinal relations among family risk factors and childhood externalizing symptoms. Developmental Psychology, 45(2), 491-502.

Bowlby, J. (1989). Uma base segura: Aplicações clínicas da teoria do apego. Porto Alegre: Artes Médicas.

Brajsa-Zganec, A. (2005). The long-term effects of war experiences on children's on the Republic of Croatia. Child Abuse \& Neglect, 29(1),33-43.

Cabrera, P., Urrutia, B., Vera, V., Alvarado, M., \& VeraVillarroel, P. (2005). Ansiedad y depresión en niños diagnosticados con cáncer. Revista de Psicopatologia y Psicologia Clinica, 10(2), 115-123.
Calderaro, R. S. S., \& Carvalho, C. V. (2005). Depressão na infância: Um estudo exploratório. Psicologia em Estudo, 10(2), 181-189.

Calvillo, M.E. N. (2004). Depresión en niños con trastorno por déficit de atención con hiperactividad. Revista Reflexiones, 83(1), 107-120.

Castro, E. K. (2003). Depressão em crianças com doenças crônicas. Aletheia, 17, 31-39.

Cole, D. A., Tram, J. M., Martin, J. M., Hoffman, K. B., Ruiz, M. D., Jacquez, F. M., et al. (2002). Individual differences in the emergence of depressive symptoms in children and adolescents: A longitudinal investigation of parent and child reports. Journal of Abnormal Psychology, 111(1), 156-165.

Coutinho, M. P. L., \& Ramos, N. (2008). Distúrbios psicoafetivos na infância e adolescência: Um estudo transcultural. Psico (PUCRS), 39(1), 23-27.

Chorney, D. B., Detweiler, M. F., Morris, T. L., \& Kuhn, B. R. (2008). The interplay of sleep disturbance, anxiety and depression in children. Journal of Pediatric Psychology, 33(4), 339-348.

Cruvinel, M., \& Boruchovitch, E. (2003). Depressão infantil: Uma contribuição para a prática educacional. Psicologia Educacional, 7(1):77-84.

Deakin, E. K., \& Nunes, M. L. T. (2008). Investigação em psicoterapia com crianças: Uma revisão. Revista de Psiquiatria do Rio Grande do Sul, 30(1). Recuperado em 20 jun.2010,em http://www. scielo.br/scielo.php?script $=$ sci_arttext\&pid $=\mathrm{S} 0101$ $-81082008000200003 \& \operatorname{lng}=$ pt\&nrm $=$ iso $>$

Dell'Aglio, D. D., \& Hutz, C. S. (2004). Depressão e desempenho escolar em crianças e adolescentes institucionalizados. Psicologia: Reflexão e Crítica, 17(3), 351-357.

Dell'Aglio, D. D., Borges, J. L., \& Santos, S. S. dos. (2004). Eventos estressores e depressão em adolescentes do sexo feminino. Psico (PUCRS), 35(1), 43-50.

Dutra, E. M. (2001). Depressão e suicídio em crianças e adolescentes. Mudanças, 9(15), 27-35.

Fensterseifer, L., \& Werlang, B. S. (2003). Suicídio na infância: Será a perda da inocência? Psicologia Argumento, 21(35), 39-46. 
Fischer-Kern, M., Tmej, A., Kapusta, N. D., Naderer, A., Leithner-Dziubas, K., Loffler-Staska, H., et al. (2008). The capacity for mentalization in depressive patients: a pilot study. Z Psychosom Med Psychoter, 54(4), 368-380.

Fonagy, P. (2003). The research agenda: The vital need for empirical research in child psychotherapy. Journal of Child Psychotherapy, 29(2), 36-129.

Fonagy, P., Target, M., Cottrell, D., Phillips, J., \& Kurtz, Z. (2002). What Works for Whom? A critical review of treatments for children and adolescents. New York: The Guilford Press.

Franco, M. H. P., \& azorra, L. (2007). Criança e luto: Vivências fantasmáticas diante da morte do genitor. Estudos de Psicologia, 24(4), 503-511.

Freud, S. (1996). Luto e melancolia. In Edição Standard Brasileira das Obras Psicológicas Completas de Sigmund Freud, (J. Salomão, trad., Vol. XIV). Rio de Janeiro: Imago. (Originalmente publicado em 1915).

Finzi, R., Ram, A., Shnit, D., Har-Even, D., Tyano, S., \& Weizman, A. (2008). Comorbid depression and ADHD in children and adolescents. Psychiatric Times, 30(6), 112-126.

Gladstone, T. R. G., \& Beardslee, W. R. (2009). The prevention of depression in children and adolescents: A review. Canadian Journal of Psychiatry, 54(4), 212-221.

Goldberg, A. E. (2007). (How) does it make a difference? Perspectives of adults with lesbian, gay, and bisexual parents. American Journal of Orthopsychiatry, 77, 550-562.

Gouveia, V. V., Barbosa, G. A., Almeida, H. J. F., \& Gaião, A. A. (1995). Inventário de depressão infantil - CDI: Estudo de adaptação com escolares de João Pessoa. Jornal Brasileiro de Psiquiatria, 44(7), 345-349.

Grote, N. K., \& Frank, E. (2003). Difficult-to-treat depression: The role of contexts and comorbidities. Biological Psychiatry, 53(8), 660-670.

Hazell, P. (2009). Depression in children and adolescents. Clinic Evidence, 7, 1-31.

Horn, H., Geiser-Elze, A., Reck, C., Hartmann, M., Stefini, A., Victor, D., et al. (2005). Praxis der Kinderpsychologie und Kinderpsychiatrie. Vandenhoeck \& Ruprecht, 54(7), 578-597.
Ito, L. M., \& Lotufo Neto, F. (2000). Teorias cognitivo-comportamentais, interpessoal e construtivista. In B. Lafer, O. P. Almeida, R. Fraguás Jr., \& E. C. Miguel. (Org.). Depressão no ciclo da vida (pp. 82-91). Porto Alegre: Artes Médicas Sul.

Jureidini, J. (2009). How do we safely treat depression in children, adolescents and young adults? Medicine National Institutes of Health, 32(4), 275-282.

Kessler, R. C., Avenevoli S., \& Merikangas K. (2001). Mood disorders in children and adolescents: An epidemiologic perspective. Biological Psychiatry, 49(12), 1002-1114.

Kim, J., \& Cicchetti, D. (2006). Longitudinal trajectories of self system and depressive symptoms among maltreated an non - maltreated children. Child Development, 77, 624-639.

Kovács, M. (1983). The children's depression inventory: A self-rated depression scale for school age youngsters. Pittsburg, PA: University of Pittsburgh, School of Medicine.

Kovács, M. (1985). The children's depression inventory (CDI). Psychopharmacology Bulletin, 21(4), 995-998.

Kovács, M. J. (1992). Children depression inventory CDI: Manual. New York: Multi-Health Systems.

Kovács, M. (2003). Children's depression inventory (CDI): Technical manual update. Toronto: MulthiHealth Systems Inc.

Kuczynski, E., Marcolin, M. A., \& Assumpção Jr., B. A. (2001). Atualização sobre o tratamento de depressão na infância e adolescência. Revista da Associação Brasileira de Psiquiatria Biológica, 45(9), 46-51.

Lakdawalla, Z., Hankin, B. L., \& Mermelstein, R. (2007). Cognitive theories of depression in children and adolescents: a conceptual and quantitative review. Child Clinical Family Psychology, 10(1), 1-24.

Lima, D. (2004). Depressão e doença bipolar na infância e adolescência. Jornal de Pediatria, 80(2), 11-20.

Margetic, B., Aukst-Margetic, B., Bilic, E., Jelusic, M., \& Tambic Bukovac, L. (2005). Depression, anxiety and pain in children with juvenile idiopathic arthritis (JIA). European Psychiatry, 20(3), 274-276. 
Mehler-Wex, C., \& Kölch, M. (2008). Depression in children and adolescents. Deutch Arztebl Int, 105(9), 149-155.

Mericangaas, K. R., \& Angst, J. (1995). The challenge of depressive disorders in adolescence. In M. Rutter (Org.). Psychosocial disturbances in young people (pp. 3-6). London: Cambridge University Press.

Michael, K. D., \& Crowley, S. L. (2002). How effective are treatments for child and adolescent depression? A meta-analytic review. Clinical Psychology Review, 22(2), 247-269.

Morrow, M. T., Hubbard, J. A., McAuliffe, M. D., Rubin, R.M., \& Dearing, K. F. (2006). Childhood aggression, depressive symptoms, and peer rejection: The mediational model revisited. International Journal of Behavioral Development, 30, 240-248.

O'Connor, M. J., \& Paley, B. (2006). The relationship of prenatal alcohol exposure and the postnatal environment of child depressive symptoms. Journal of Pediatric Psychology, 31(1), 50-64.

Organização Mundial da Saúde-OMS. (1993). Violence and health. Geneva. Suiça: World Health Statistics Quarterly do World Health Organization.

Parreira, S. M. C. P., \& Justo, J. S. (2005). A criança abrigada: Considerações acerca do sentido da filiação. Psicologia em Estudo, 10(2), 175-180.

Perera, H. (2008). Depression in children and adolescents. Ceylon Medical Journal, 53(2), 65-67.

Ramires, V. R. R., Benetti, S. P. C., Silva, F. J. L., \& Flores, G. G. (2009). Saúde Mental de Crianças no Brasil: Uma revisão de literatura. Interação em Psicologia, 13(2), 311-322.

Reis, R. L. R., \& Figueira, I. L. V. (2001). Transtorno depressivo na clínica pediátrica. Revista Pediatria Moderna, 37, 212-222.

Reppold, C. T., \& Hutz, C. S. (2003). Exigência e responsividade parental como preditores de depressão em adolescentes no sul do Brasil. Avaliação Psicológica, 3, 175-184.

Ribeiro, K. C. S., Oliveira, J. S. C., Coutinho, M. P. L., \& Araújo, L. F. (2007). Representações sociais da depressão no contexto escolar. Paidéia, 17(38), 417-430.
Rodrígues de Cossío, A., \& Granada Jiménez, O. (2007). Transtornos depresivos em la infancia y adolescencia. RevistaClínicade Medicina de Familiar, 1(6), 270-276.

Rotondaro, D. P. (2002). Os desafios constantes de uma psicóloga no abrigo. Psicologia: Ciência e Profissão, 22(3), 8-13.

Runyon, M. K., Faust, J., \& Ovaschel,H. (2002). Differential symptom pattern of post-traumatic stress disorder in maltreated children with and without concurrent depression. Child Abuse e Neglect, 26, 39-53.

Scivoletto, S., \& Tarelho, L. G. (2002). Depressão na infância e adolescência. Revista Brasileira de Medicina, 59(8), 555-557.

Son, S. E., \& Kirchner, J. T. (2000). Depression in children and adolescents. American Family Physician, 62(10), 2297-2308.

Suveg, C., Hoffman, B., \& Thomassin, K. (2009). Common and specific emotion-related predictors of annnxious and depressive symptoms in youth. Child Psychiatry Human Development, 40(2), 223-239.

Trevisan, J. (2004). Psicoterapia psicanalítica e depressão de difícil tratamento: À procura de um modelo integrador. Revista de Psiquiatria, 26(3), 319-328.

Varley, C. K. (2006). Treating depression in children and adolescents: what options now? Medicine National Institutes of Health, 20(1), 1-13.

Versiani, M., Reis, R., \& Figueira, I. (2000). Diagnóstico do transtorno depressivo na infância e adolescência. Revista de Psiquiatria, 10-12(49), 367-382.

Wagner, K. D., Jonas, J., Findling, R. L., Ventura, D., \& Saikali, K. (2006). A double-blind, ramdomized, placebo-controlled trial of escitalopran in the treatment of pediatric depression. Journal of the American Academy of Child and Adolescent Psychiatry, 45(3), 280-288.

Wasliick, B., Schoenholz, D., \& Pizarro, R. (2003). Diagnosis and treatment of chronic depression in children and adolescents. Journal Psychiatric Practice, 9(5), 345-366.

Watanabe, N., Hunot, V., Omori, I. M., Churchill, R., \& Furukawa, T. A. (2007). Psychotherapy for depression among children and adolescents: a systematic review. Acta Psychiatrica Scandinavica, 116(2), 84-95. 
Wathier, J. L., \& Dell'Aglio, D. D. (2007). Sintomas depressivos e eventos estressores em crianças e adolescentes no contexto de institucionalização. Revista Psiquiátrica, 29(3), 305-314.

Wathier, J. L., Dell'Aglio, D. D., \& Bandeira, D. R. (2008). Análise fatorial do inventário de depressão infantil (CDI) em amostra de jovens brasileiros. Avaliação Psicológica, 7(1), 75-84.

Weisz, J. R., McCarty, C. A., \& Valeri, S. M. (2006). Effects of psychotherapy for depression in children and adolescents: A meta-analysis. Psychological Bulletin, 132(1), 132-149.

West, A., \& Salmon, G. (2000). Bullying and depression: A case report. International Journal of Psychiatry in Clinical Practice, 4(1), 73-75.

Zavaschi, M. L. S., Satler, F., Poester, D., Vargas, C. F., Piazenski, R., Rohde, L. A. P., et al. (2002). Associação entre trauma por perda na infância e depressão na vida adulta. Revista Psiquiátrica, 24(4), 189-195.

Recebido: 29/06/2010

Received: 06/26/2010

Aprovado: 03/09/2010

Approved: 09/03/2010 